\title{
SCHRÖDINGER OPERATORS WITH POTENTIAL WAVEGUIDES ON PERIODIC GRAPHS
}

\author{
Olaf Post and Natalia Saburova
}

\begin{abstract}
We consider discrete Schrödinger operators with periodic potentials on periodic graphs perturbed by guided positive potentials, which are periodic in some directions and finitely supported in other ones. The spectrum of the unperturbed operator is a union of a finite number of non-degenerate bands and eigenvalues of infinite multiplicity. It is known that the spectrum of the perturbed operator consists of the spectrum of the unperturbed one and the additional guided spectrum, which is also a union of a finite number of bands. We estimate the positions of the guided bands in gaps of the unperturbed operator in terms of eigenvalues of Schrödinger operators on some finite graphs. We also determine sufficient conditions for the guided potentials under which the guided bands do not appear in gaps of the unperturbed problem.
\end{abstract}

Mathematics subject classification (2010): 47A10, 47A58, 47A75.

Keywords and phrases: Discrete Schrödinger operator, periodic graph, guided waves, spectral gaps.

\section{REFERENCES}

[1] S. Alama, P.A. Deift, R. Hempel, Eigenvalue branches of the Schrödinger operator $H-\lambda W$ in a spectral gap of H, Comm. Math. Phys. 121 (1989), 291-321.

[2] K. ANDO, Inverse scattering theory for discrete Schrödinger operators on the hexagonal lattice, Ann. Henri Poincaré 14 (2013), 347-383.

[3] K. Ando, H. Isozaki, H. Morioka, Spectral properties of Schrödinger operators on perturbed lattices, Ann. Henri Poincaré 17 (2016), 2103-2171.

[4] J. ARAZY, L. ZeLENKo, Finite-dimensional perturbations of self-adjoint operators, Integral Equations Operator Theory 34, 2 (1999), 127-164.

[5] M. Sh. Birman, Discrete spectrum in the gaps of a continuous one for perturbations with large coupling constant, Adv. Soviet Math. 7 (1991), 57-73.

[6] J. Blank, P. ExNer, M. HaVliceK, Hilbert Space Operators in Quantum Physics, Springer Science and Business Media B.V., 2008.

[7] A. H. Castro Neto, F. Guinea, N. M. R. Peres, K.S. Novoselov, A. Geim, The electronic properties of graphene, Rev. Mod. Phys. 81 (2009), 109-162.

[8] P. A. DeIfT, R.Hempel, On the existence of eigenvalues of the Schrödinger operator $H-\lambda W$ in a gap of $\sigma(H)$, Comm. Math. Phys. 103 (1986), 461-490.

[9] J. S. Fabila-Carrasco, F. Lledó, O. Post, Spectral gaps and discrete magnetic Laplacians, Linear Algebra Appl. 547, 15 (2018), 183-216.

[10] R. Halir, P.J. Bock, P. Cheben, A. Ortega-Moñux, C. Alonso-Ramos, J.H. Schmid, J. Lapointe, D.-X. Xu, J. G. Wanguemert-PereZ, I. Molina-FernandeZ, S. JanZ, Waveguide sub-wavelength structures: a review of principles and applications, Laser Photon. Rev. 9 (2015), 25 49.

[11] P. HarRis, Carbon nano-tubes and related structure, Cambridge, Cambridge University Press, 2002.

[12] W.A. Harrison, Electronic Structure and the Properties of Solids: The Physics of the Chemical Bond, Dover Publications, Inc., New York, 1989.

[13] R. HemPel, Eigenvalues in gaps and decoupling by Neumann boundary conditions, J. Math. Anal. Appl. 169 (1992), 229-259. 
[14] R. HEMPEL, Eigenvalues of Schrödinger operators in gaps of the essential spectrum - an overview, Contemp. Math. 458 (2008), 393-407.

[15] Y. Higuchi, T. Matsumoto, O. Ogurisu, On the spectrum of a discrete Laplacian on $\mathbb{Z}$ with finitely supported potential, Linear and multilinear algebra 59, 8 (2011), 917-927.

[16] Y. Higuchi, Y. Nomura, Spectral structure of the Laplacian on a covering graph, European J. Combin. 30, 2 (2009), 570-585.

[17] Y. HigUCHI, T. SHIRAI, The spectrum of magnetic Schrödinger operators on a graph with periodic structure, J. Funct. Anal. 169 (1999), 456-480.

[18] Y. HigUCHI, T. ShIRAI, A remark on the spectrum of magnetic Laplacian on a graph, the proceedings of TGT10, Yokohama Math. J. 47 (1999), Special issue, 129-142.

[19] Y. HIgUCHI, T. SHIRAI, Weak Bloch property for discrete magnetic Schrödinger operators, Nagoya Math J. 161 (2001), 127-154.

[20] Y. HigUCHI, T. SHIRAI, Some spectral and geometric properties for infinite graphs, AMS Contemp. Math. 347 (2004), 29-56.

[21] F. Hiroshima, I. Sasaki, T. Shirai, A. Suzuki, Note on the spectrum of discrete Schrödinger operators, J. Math-for-Industry 4 (2012), 105-108.

[22] R. Horn, C. Johnson, Matrix analysis, Cambridge University Press, 1985.

[23] H. IsOZAKI, E. KOROTYAEV, Inverse problems, trace formulae for discrete Schrödinger operators, Ann. Henri Poincaré 13 (2012), 751-788.

[24] S. G. Johnson, P. R. Villeneuve, S. Fan, J. D. JoAnnopoulos, Linear waveguides in photonic crystal slabs, Phys. Rev. B 62 (2000), 8212-8222.

[25] S. G. Johnson, J. D. JoAnnopoulos, Photonic crystals. The road from theory to practice, Springer US, 2002.

[26] E. Korotyaev, N. Saburova, Schrödinger operators on periodic discrete graphs, J. Math. Anal. Appl. 420, 1 (2014), 576-611.

[27] E. Korotyaev, N. Saburova, Spectral band localization for Schrödinger operators on periodic graphs, Proc. Amer. Math. Soc. 143 (2015), 3951-3967.

[28] E. Korotyaev, N. Saburova, Schrödinger operators with guided potentials on periodic graphs, Proc. Amer. Math. Soc. 145, 11 (2017), 4869-4883.

[29] E. Korotyaev, N. Saburova, Magnetic Schrödinger operators on periodic discrete graphs, J. Funct. Anal., 272 (2017), 1625-1660.

[30] E. Korotyaev, N. Saburova, Invariants for Laplacians on periodic graphs, Math. Ann., 377 (2020), 723-758.

[31] A. Kutsenko, Wave propagation through periodic lattice with defects, Comput. Mech. 54 (2014), $1559-1568$.

[32] A. Kutsenko, Algebra of multidimensional periodic operators with defects, J. Math. Anal. Appl. 428, 1 (2015), 217-226.

[33] A. KutsenKo, Algebra of $2 D$ periodic operators with local and perpendicular defects, J. Math. Anal. Appl. 442, 2 (2016), 796-803.

[34] K.S. Novoselov, A.K. Geim ET AL, Electric field effect in atomically thin carbon films, Science 306, 5696 (2004), 666-669.

[35] G.G. Osharovich, M.V. Ayzenberg-Stepanenko, Wave localization in stratified square-cell lattices: The antiplane problem, J. Sound Vib. 331 (2012), 1378-1397.

[36] V.S. Rabinovich, S. Roch, Essential spectra of difference operators on $\mathbb{Z}^{n}$-periodic graphs, J. Phys. A 40, 33 (2007), 10109-10128.

[37] M. ReEd, B. Simon, Methods of modern mathematical physics, vol.I. Functional analysis, Academic Press, New York, 1980.

[38] M. ReED, B. Simon, Methods of modern mathematical physics, vol.IV. Analysis of operators, Academic Press, New York, 1978.

[39] G. TeSCHL, Mathematical Methods in Quantum Mechanics with Applications to Schrödinger Operators, American Mathematical Society, Graduate Studies in Mathematics, Volume 99, 2009. 